\title{
Run-to-Run Control of DC-Sputtering Processes
}

\author{
by \\ Denis Gillet ${ }^{1}$, Oscar D. Crisalle ${ }^{2}$, and Dominique Bonvin ${ }^{1}$ \\ ${ }^{1}$ Swiss Federal Institute of Technology, CH - 1015 Lausanne, Switzerland \\ ${ }^{2}$ University of Florida, Gainesville, FL 32611, USA
}

\begin{abstract}
A new nonlinear model is proposed for describing the relationship between the relevant inputs and outputs of a DCmagnetron sputtering system used in a manufacturing line for digital-compact discs and other optical data-storage devices. It is shown that the process is intrinsically discrete, and that it has an inherent transport delay. A control scheme is proposed consisting of a Smith predictor to address the delayed dynamics, and a globally linearizing operator introduced to address the nonlinear nature of the model. The control structure requires knowledge of two model parameters, namely, the maximum film reflectivity and the characteristic-energy factor of the sputterer, and makes use of one tuning variable, namely, the parameter of an integral-only controller. The performance of the control system is illustrated via a simulation study.
\end{abstract}

\section{Introduction}

Sputtering is a process used for removing material from a target source through bombardment with energetic ions. The material ejected from the target falls onto a substrate where it forms a film. When the target material is a conductor, a DC power source can be used to generate the energy needed to ionize the molecules of a gas via a glow discharge. The substrate is typically placed over the anode. The gas ions are accelerated towards the target (which acts as the cathode) where upon impact they "sputter" atoms off the source [1] [2] [3].

The deposition rate of a DC sputtering system can be greatly increased by the use of magnetic fields that intensify the gas discharge [1]. Such DC-magnetron sputtering systems are commonly used in the microelectronics industry to deposit aluminum films that serve as circuit interconnections, and in the manufacture of digital video discs (commonly referred to as DVDs) as well as digital compact discs (CDs) to deposit reflective aluminum films on write-only CDs or reflective gold films on read-write CDs. Figure 1 shows the elements of a DC-magnetron sputtering system used to deposit a gold film on a CD. A solid-gold target is used as the cathode, while a $\mathrm{CD}$ positioned over the anode serves as the substrate.
The number of atoms sputtered from the target by each impacting ion is known as the sputtering yield, which is a property of each material and of the energy of the ions. The rate of deposition of gold or aluminum metal on the substrate is a function of both the sputtering yield and the ion current.

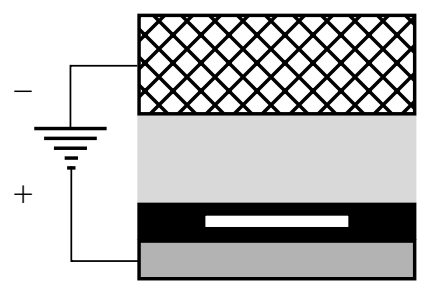
Gold target
(cathode)

Argon gas

CD substrate

Anode

Figure 1. DC-magnetron sputtering system for coating a $\mathrm{CD}$ with a gold film.

Figure 2 shows the arrangement of a typical manufacturing line for coating CDs with an aluminum or gold film. The process consists of four chambers arranged in sequential order, namely, a sputtering, a cooling, a coating, and a measuring chamber. The metal film is deposited in the sputtering chamber which houses a DC-magnetron system. Then the CD is promoted to the cooling chamber, where its temperature is reduced to ambient conditions. Next, the coating chamber is used to deposit an extremely thin antireflective film and to inject specialized color markings. Finally, the CD is loaded into the measuring chamber which houses an optical device that measures the reflectivity of the film. Each chamber has an admission queue consisting of several CDs that are waiting to be processed in their order of arrival.

At the measuring station (located at the far right of Figure 2), an automated system measures the reflectivity $R$ of the film deposited on the $\mathrm{CD}$. The measuring chamber has an admission queue that consists of several CDs (two CDs in the case of the process depicted in Figure 2). When the measurement process for a $\mathrm{CD}$ is completed, the $\mathrm{CD}$ is removed from the measuring chamber by a robotic arm, the first $\mathrm{CD}$ in the measuring queue is loaded into the measuring chamber, and the second $\mathrm{CD}$ in the measuring queue is promoted to the first position in the queue via a conveyor belt. At this time a $\mathrm{CD}$ is removed from the coating chamber and loaded in the last

\footnotetext{
* Author to whom all correspondence should be sent (crisalle@che.ufl.edu, phone 352-392-5120, FAX 352-392-9513).
} 
position of the measuring queue. Analogous operations of loading, processing, and unloading of CDs occur at each chamber, following a synchronized cascade of activities. The length of the admission queues for each of the chambers is designed as a function of the throughput (number of CDs processed per unit time) of each chamber, and whether the chamber can simultaneously process several CDs. The sputtering chamber is typically a single-CD system. The manufacturing process can therefore be viewed as a series of sequential batch operations.

All process control actuations are carried out in the sputtering chamber, where an operator specifies the power $P$ of the DC power supply, and the duration $t_{s}$ of the sputtering cycle. The objective of the process is to obtain films with a given reflectivity, which is typically expressed as a percentage of the maximum reflectivity of the film. If the reflectivity is too low, the CD will not perform adequately because digital information is extracted from the CD by detecting the reflection of a laser beam focused on the metallic film. On the other hand, if the reflectivity is too high, the performance of the $\mathrm{CD}$ is not affected; however, the sputtering process has deposited more metal than necessary, resulting in additional production costs which can be particularly significant when sputtering gold films.

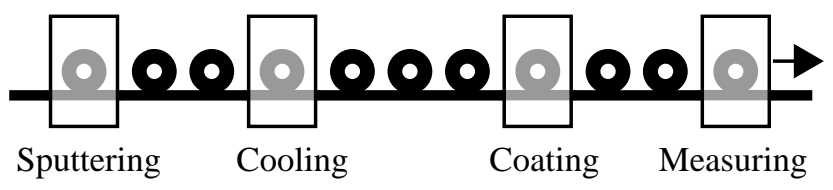

Figure 2. Typical processing sequence used for depositing metal films on CDs. The CDs are sequentially exposed to sputtering, cooling, coating, and measuring operations in a series of four chambers.

\section{Experimental Modeling Approach}

Experimental studies were carried out using an aluminum DC-magnetron sputtering system to characterize the relationship between the measured variable (i.e., the reflectivity $R[\%]$ ) and manipulated variables (i.e., the sputtering power $P[\mathrm{~kW}]$ and the duration of the sputtering cycle $\left.t_{s}[\mathrm{~s}]\right)$. A total of $106 \mathrm{CDs}$ were sputtered at various values of power and cycle duration. The resulting reflectivity was measured for each CD. The maximum reflectivity $R_{\max }$ attainable by aluminum films deposited in this particular DC-magnetron system was also determined experimentally using the largest DC power possible, and the longest cycle time that was deemed acceptable in the manufacturing environment. The sputtering energy $E=P t_{s}[\mathrm{~kW} \mathrm{~s}]$ for each experiment was calculated, and the corresponding maximum sputtering energy $E_{\text {max }}$ was readily determined.

The experimental results are shown in Figure 3, where the reflectivity ratio $R / R_{\max }$ is plotted as a function of the sputter- ing-energy ratio $E / E_{\text {max }}$. Analysis of the data reveals that, for any choice of source power $P$ and cycle duration $t_{s}$, the resulting reflectivity is determined by the value of the sputtering-energy ratio $E / E_{\max }$ that therefore plays the role of a process invariant. Hence, for process control purposes, there is only one relevant manipulated variable, namely, the sputtering-energy ratio. This is a useful observation for the manufacturer, who typically would prefer to specify the cycle duration independently in order to meet production throughput constraints and maintain the cadence of the manufacturing line.

\subsection{Static Model for DC-Magnetron Sputtering}

Figure 3 suggests that the experimental data can be adequately represented by the nonlinear static relationship

$$
\frac{R}{R_{\max }}=1-e^{-\frac{1}{\xi} \frac{E}{E_{\max }}}
$$

where $R_{\text {max }}$ is the maximum reflectivity of the sputtered film, and $\xi$ is the characteristic-energy factor, a parameter that depends on the specific geometry of the sputterer as well as

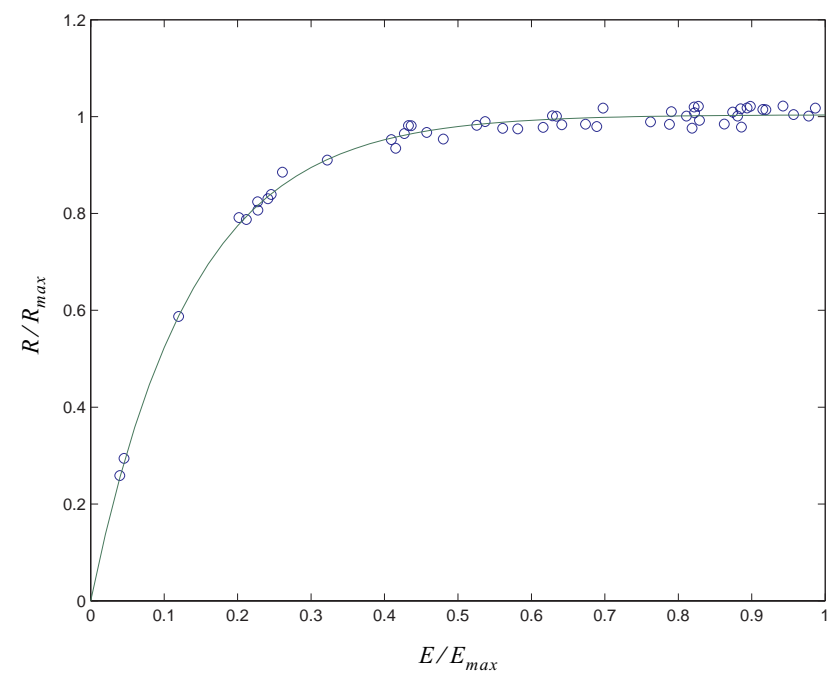

Figure 3. Experimentally determined values of normalized reflectivity versus normalized sputtering power. The circles represent the experimental data and the continuous line represents the model proposed by the authors.

on the nature of the target and the level of target load. The variable $R / R_{\max }$ is known as the reflectivity ratio. Parameters $R_{\max }$ and $\xi$ can be readily determined from the experimental data using standard least-squares regression techniques [4]. To the best of the authors' knowledge, the static model (1) has not been previously identified in the literature as an effective structure for relating the measured output to the manipulated inputs in DC-magnetron sputtering systems. 


\subsection{Dynamic Model for DC-Magnetron Sputtering}

Note that in the process represented in Figure 2, the film reflectivity is measured on a CD while at the same time a sputtering operation is being carried out upstream on another CD. Therefore, the process has an inherent transport delay (or dead time). This introduces a time-delay between the sputtering control actuation and the measurement. Although the time elapsed during transport may vary depending on the throughput of each processing chamber, the number of CDs located between the sputtering and measuring chambers remains constant. Let $k$ denote a CD counter located at the sputtering station, such that it increases by one each time a CD leaves the sputtering station. Using the CD counter as a processing reference index, it follows that the reflectivity $R(k)$ of the $k$ th -processed CD depends on the energy $E(k)$ applied when it was in the sputtering chamber. Then equation (1) can be written in the form

$$
\frac{R(k)}{R_{\max }}=1-e^{-\frac{1}{\xi} \frac{E(k)}{E_{\max }}}
$$

According to the proposed indexing procedure, $k-1$ represents the last $\mathrm{CD}$ in the cooling-admission queue, $k-2$ the next-to-last $\mathrm{CD}$, and so on. The index $k$ can also be interpreted as a batch or run index.

Let $d$ denote the total number of CDs located in the cooling, coating, and measuring stations, and in their respective admission queues. It then follows that the sequential manufacturing process of Figure 2 has a time-delay equal to $d$. Let $y(k)$ denote the measured reflectivity ratio value obtained at the measuring station while the $k-t h \mathrm{CD}$ is being processed in the sputtering chamber. Since a total of $d$ CDs lie in the chain between the sputtering station to the measuring station, the measurement $y(k)$ taken at the measuring station when the counter value is equal to $k$ is in fact the reflectivity ratio of the metal film corresponding to a CD sputtered when the counter value was equal to $k-d$; equivalently

$$
y(k)=\frac{R(k-d)}{R_{\max }}
$$

Combining equations (2) and (3) yields the final input-output nonlinear process model

$$
y(k)=1-e^{-\frac{1}{\xi} \frac{E(k-d)}{E_{\max }}}
$$

where the delay $d$ is exactly known. It is important to note that the representation (4) is a discrete-time model. In this case there is no sampling period involved, and the index $k$ is not necessarily an indicator of "clock-on-the-wall" time. Note that the manufacturing process may be stopped for maintenance or repair operations for any given duration of time without affecting the value of the index counter.

\section{Control Strategy}

The control design method proposed consists of first introducing a globally linearizing transformation for the dynamic model (4), to obtain a static linear system with an exactly known delay. A suitable classical linear controller is then implemented via a Smith predictor scheme [5][6][9]. Details are given below.

\subsection{Global Linearization}

An inverse-model can be used in this case to globally linearize the nonlinear static process model. Advancing equation (4) by $d$ samples yields

$$
y(k+d)=1-e^{-\frac{1}{\xi} \frac{E(k)}{E_{\max }}} \equiv v(k)
$$

where we recognize $E(k)$ as the primary input variable. This equation also introduces the definition of the intermediate input variable $v(k)$ that is simply related to $E(k)$ through the inverse model

$$
E(k)=-\xi E_{\text {max }} \ln (1-v(k))
$$

obtained after rearranging terms in (5) and taking the natural logarithm. Shifting indices in (5) yields the linear discrete model

$$
y(k)=v(k-d)
$$

which features a pure delay $d$. Therefore, the introduction of the inverse-model map (6) effectively causes the global linearization of the original nonlinear open-loop model (4).

Since the intermediate manipulated variable $v(k)$ is in fact a reflectivity ratio, it must be constrained to satisfy the inequality

$$
0 \leq v(k)<1
$$

where the lower limit is introduced to require that the reflectivity ratio be nonnegative, and the upper bound is imposed to recognize that the reflectivity ratio cannot be greater than one and to prevent a singularity in the argument of the natural-logarithm function in (6).

\subsection{Feedback Control via a Smith Predictor Design}

Since the global linearization procedure yields a pure-delay discrete-time model, it is natural to consider a Smith predictor design for the control system. Given that the Smith predictor scheme is free from its known robustness deficiencies when there is uncertainty in the time delay [7][9], it is particularly appealing to apply it to the sputtering control problem since the delay is accurately known.

The main task of the Smith predictor is to use feedback to adjust the intermediate input variable $v(k)$ in order to track a user-defined reflectivity ratio set point $y_{r}(k)$. In order to satisfy the saturation constraints (8), let the output of the Smith 
predictor $u(k)$ be related to the intermediate manipulated variable through the saturation assignment

$$
v(k)= \begin{cases}0 & \text { if } u(k)<0 \\ u(k) & \text { if } 0 \leq u(k)<1 \\ 1-\varepsilon & \text { otherwise }\end{cases}
$$

where $\varepsilon$ is a small positive number. As is the usual practice, the Smith predictor design is carried out under the assumptions that the inverse-model (6) is globally linearizing, and that the saturation constraints are inactive (i.e., $v(k)=u(k)$ ). Under these conditions the open-loop model reduces to

$$
y(k)=u(k-d)
$$

In order to introduce deviation variables and to invoke the $\mathrm{z}$ Transform, consider a steady-state operating point $\left(y_{0}, u_{0}\right)$ of (10), which is trivially determined as $y_{0}=u_{0}$ where $u_{0}$ is an arbitrary reflectivity bias corresponding to a steadystate value of the sputtering energy. Introducing the deviation variables $\tilde{u}(k)=u(k)-u_{0}$ and $\tilde{y}(k)=y(k)-y_{0}$ the model (10) can be rewritten in the form

$$
\tilde{y}(k)=\tilde{u}(k-d)
$$

Let the variables $Y(z)$ and $U(z)$ respectively denote the ztransforms of $\tilde{y}(k)$ and $\tilde{u}(k)$. Furthermore, let the deviationform of the set-point variable be given by $\tilde{y}_{r}(k)=y_{r}(k)-y_{0}$ and its associated z-transform $Y_{r}(z)$. Then the linear model (11) can be written as $Y(z)=z^{-d} U(z)$. The structure of the Smith predictor scheme for this process is shown in Figure 4, where the synthesis task is to find a suitable controller $C(z)$.

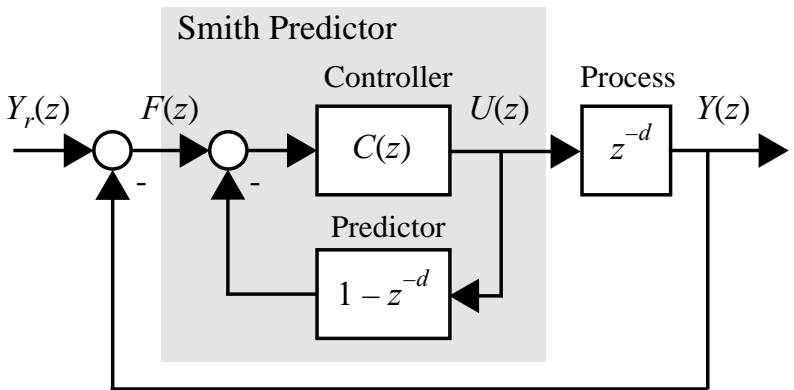

Figure 4. Structure of the Smith predictor used for designing the controller $C(z)$ under the assumptions of exact global linearization and inactive constraints.

A controller can be easily realized by first recognizing that the closed-loop transfer function in Figure 4 is $Y(z) / Y_{r}(z)=z^{-d} C(z) /(1+C(z))$, and then requiring that the non-delayed closed-loop adopt a first-order structure by setting

$$
\frac{C(z)}{1+C(z)}=(1-\alpha) \frac{z}{z-\alpha}
$$

where the real parameter $-1<\alpha<1$ represents a userselected closed-loop pole. Note that the prescribed ranges for the parameter $\alpha$ ensure the stability of the closed loop. In order to avoid ringing and dead-beat behavior, the pole is further restricted to be in the range $0<\alpha<1$. Solving (12) for $C(z)$ yields the integral-only controller

$$
C(z)=\frac{1}{\tau_{i}} \frac{z}{z-1}
$$

where the dimensionless integral-control constant $\tau_{i}$ is related to the desired closed-loop pole via the expression $\tau_{i}=\alpha /(1-\alpha)$. Therefore, the tuning of the integral-only controller (13) reduces to selecting a preferred speed of closed-loop response via adjustment of the pole $\alpha$.

Note also that the relationship between the control output $U(z)$ and the feedback error $F(z)$ is given by the transfer function

$$
U(z)=\frac{1}{\left(1+\tau_{i}\right)-\tau_{i} z^{-1}-z^{-d}} F(z)
$$

which can be inverted to yield the control equation

$$
\tilde{u}(k)=\frac{1}{1+\tau_{i}}\left[\tau_{i} \tilde{u}(k-1)+\tilde{u}(k-d)+\tilde{f}(k)\right]
$$

where $f(k)=\tilde{y}_{r}(k)-\tilde{y}(k)=y_{r}(k)-y(k)$ is the feedback error.

\subsection{Implementation Details}

Figure 5 shows the complete implementation scheme for the control structure proposed to carry out run-to-run control of the DC-magnetron sputtering process. The control system contains three major elements, namely, a Smith-predictor block, an input-saturation block, and a global-linearization block. Note that the process is characterized by a measurement delay $d$, and by a sputtering model of the form (2). The global linearizer is designed using the available process parameters $R_{\max }$ and $\xi$, which may differ from the actual process parameters $R_{\max }$ and $\bar{\xi}$ due to modeling errors. Exact linearization occurs when $R_{\max }=R_{\max }$ and $\xi=\bar{\xi}$.

Given that the system has input saturation, the integral-only controller $C(z)$ must be supplemented with an algorithm to introduce anti-reset windup protection. The procedure can be implemented using a number of standard approaches [10]. Further details are omitted here for brevity.

The injection of the constant bias $u_{0}$ as shown in Figure 5 is needed to account for the fact that the Smith predictor design is done in terms of deviation variables. In addition, given that the manufacturing system has an inherent delay $d$, no measurement feedback is available until the first sputtered CD advances through the entire post-sputtering chain and reaches the measuring chamber. As a consequence, the integral- 
only controller must remain turned off during an initial startup period of $d$ runs. This is accomplished by setting $u(k)=u_{0}$ for $k<d$.

\subsection{Complete Control Algorithm}

The overall control scheme of Figure 5 is implemented in a sequential (i.e., run-to-run) fashion. At the instant when run $k$ exits the sputtering chamber, proceed to execute the following steps:

1. Measure the reflectivity $y(k)$ currently available at the measuring station.

2. Compute the feedback error $f(k)=y_{r}(k)-y(k)$.

3. Compute the Smith predictor output $\tilde{u}(k)$ from (15).

4. Add the control bias through $u(k)=u_{0}+\tilde{u}(k)$.

5. Compute the intermediate input variable $v(k)$ from the saturation assignment (9).

6. Compute the primary input variable $E(k)$ from the inverse model (6).

7. Load run $k+1$ into the sputtering chamber and process it using the sputtering energy $E(k)$ calculated in Step 6.

During startup (i.e., $k<d$ ) the algorithm is modified by omitting Steps 1 through 3, and substituting $u(k)=u_{0}$ in Step 4. The rest of the steps remains unaltered. The complete control algorithm can be executed effectively with very modest processing hardware requirements.

\section{Simulation Study}

A simulation study is carried out for a DC-magnetron sputtering process with parameters $R_{\max }=0.80$ (i.e., the maximum reflectivity is $80 \%$ ) and $\bar{\xi}=0.20$, and with a postsputtering processing chain that introduces a measurement delay $d=10$. A start-up and tracking experiment is designed as follows: ( $i$ ) a first CD (run $k=0$ ) is processed in the sputterer while all the post-sputtering queues and chambers are empty, and (ii) the reference reflectivity is changed at run $k=15$ from $0.40 R_{\max }$ to $0.70 R_{\max }$.
The control system is implemented following the scheme shown in Figure 5, using an integral-control constant $\tau_{i}=0.5$ (i.e., $\alpha=1 / 3$ ). It is also assumed that the experimental process parameters are free from error, i.e., $R_{\max }=R_{\max }$ and $\xi=\bar{\xi}$. The upper-bound on the saturation-constraint (9) is set by choosing $\varepsilon=0.01$.

The observed trajectory of the measured reflectivity ratio $y(k)$ (dimensionless) is plotted along with the reference reflectivity ratio $y_{r}(k)$ in Figure $6 \mathrm{a}$. Figure $6 \mathrm{~b}$ shows the normalized manipulated variable $E(k) / E_{\text {max }}$, namely, the runto-run sputtering-energy adjustments made through the power supply of the sputterer. When the step-change in reference reflectivity is made for run $k=15$, the control system responds introducing a sequence of changes in the sputtering energy, as shown in Figure 6b. Due to the process delay, the effect of these manipulations is observed at the measuring station 10 runs later, as shown in Figure 6a. The net result is a fairly rapid rise towards the reference trajectory. Approximately 15 CDs are sputtered with off-target material during the tracking transient; a subset of these products will need to be discarded as reject material. Additional simulation studies using the more aggressive integral-constant setting $\tau_{i}=0.01(\alpha=0.0099)$ show that the resulting response is almost deadbeat, a desirable feature that leads to a significant reduction in reject product, but obtained at the expense of developing higher sensitivity to measurement noise.

For comparison purposes, the simulation study is repeated using an integral-only controller with parameter $\tau_{i}=2.2$ obtained through trial-and-error tuning studies. The control configuration used is obtained from Figure 5 after removing the predictor block and the global-linearizer block. The controller had an identical start-up policy to that described above. The results are shown in Figure 7, where the trajectory of the normalized reflectivity shows significant deviations from the reference values. The simulation study shows that approximately $42 \mathrm{CDs}$ are sputtered with off-target metal films during the tracking transient. In spite of significant effort invested in fine tuning the integral-only controller, the final performance is much less satisfactory than that of the

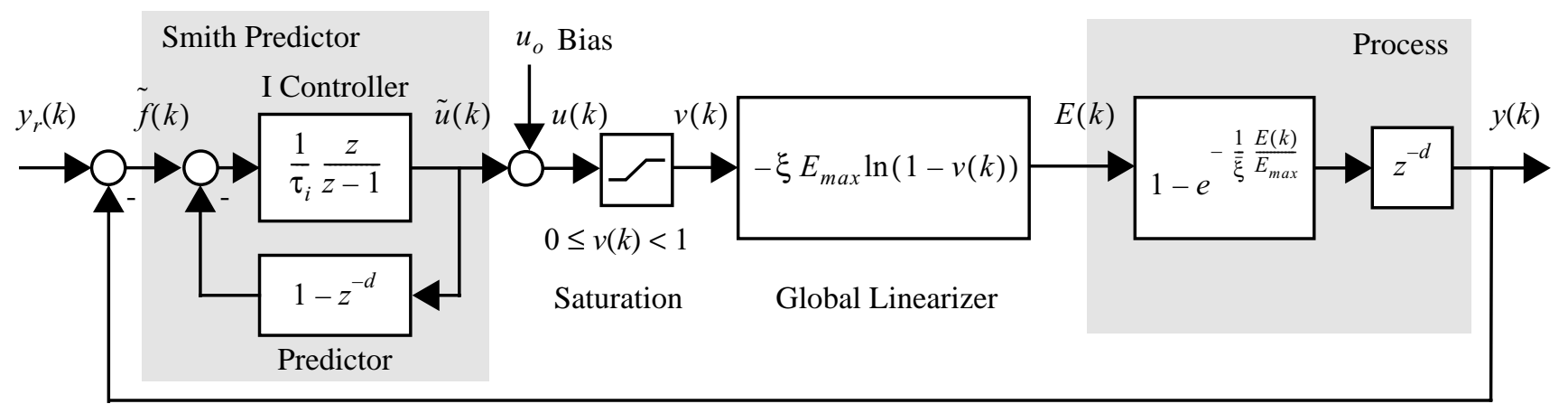

Figure 5. Overall control structure consisting of a Smith-predictor block with an integral-only controller, inputsaturation constraints, and a global linearizer. 
Smith-predictor/global-linearizer control scheme.
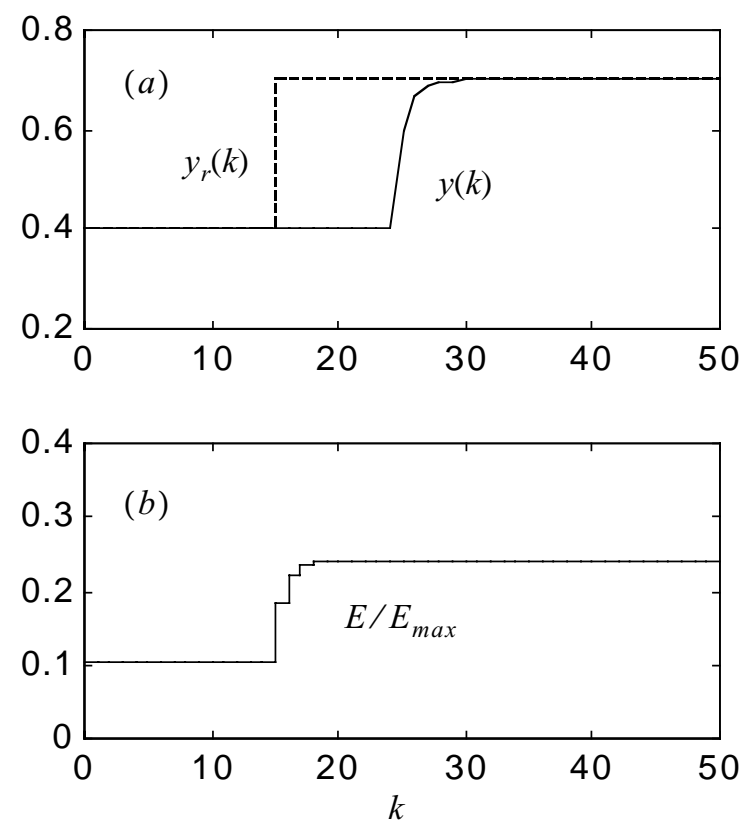

Figure 6. Closed-loop response using a Smith predictor control scheme: (a) reference trajectory and measured reflectivity ratio, (b) run-to-run adjustment of the normalized sputtering energy.

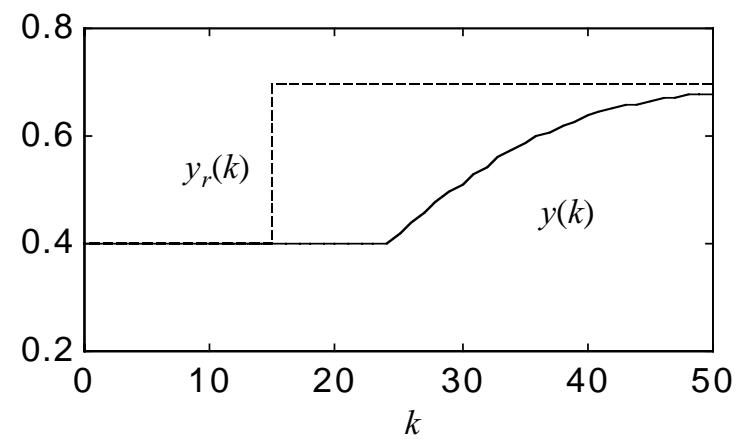

Figure 7. Closed-loop response with an integral-only controller in the absence of both a Smith-predictor and of a global linearizer.

The robustness of the proposed scheme has been studied via simulation for the case where there is plant-model mismatch (i.e., $\xi \neq \bar{\xi}$ ). It has been found that for the case where the modeling error is bound by $\xi / \bar{\xi} \leq 4.1$, the sputtering-energy variable $E(k)$ avoids saturation and delivers adequate tracking. These results suggest that acceptable performance can be obtained in spite of the presence of significant modeling errors.

\section{Concluding Remarks}

A key contribution of this work is the elucidation of a static input-output model for DC-magnetron sputtering operations.
The inherently discrete nature of the process is effectively addressed by the introduction of a time-independent index counter that tracks the evolution of the dynamics of the manufacturing process. The Smith-predictor design is effective in this case given that the transport delay is exactly known. Simulation studies suggest that the overall control scheme is capable of handling significant modeling errors, adding to the appeal of the proposed approach.

\section{References}

[1] Coclaser, Roy A., Microelectronics: Processing and Device Design, John Wiley and Sons, NY (1980).

[2] Smith, D. L., Thin-Film Deposition: Principles and Practice, McGraw-Hill, NY (1995).

[3] McDaniel, T. W.; and R. H. Victora, Handbook of Magneto-Optical Data Recording: Materials, Subsystems, Techniques, Noyes Publications, Westwood CA (1997).

[4] Box, E. P.; W. G. Hunter, and J. Stuart Hunter, Statistics for Experimenters: an Introduction to Design, Data Analysis, and Model Building, Wiley, NY (1978).

[5] Smith, O. J. M.; "Closer Control of Loops with Dead Time", Chem. Eng. Prog., 53, 217, (1957).

[6] Levine, W. S.; The Control Handbook, CRC Press Inc. and IEEE Press, (1996).

[7] Palmor, Z. J.; "Stability Properties of Smith DeadTime Compensator Controllers", Int. J. Control, 32, 6, 937-949, (1980).

[8] Khalil, H. K., Nonlinear Systems, Macmillan Pub. Co., NY (1992).

[9] Palmor, Z. J.; and Powers, D. V.; "Improved DeadTime Compensator Controllers", AIChE J., 31, 2, 215-221, (1985).

[10] Seborg, D. E.; Edgar, T. F.; and Mellichamp, D. A.; Process Dynamic and Control, Wiley New York, (1989).

\section{Acknowledgements}

The second author gratefully acknowledges support received from the National Science Foundation under grants number CTS 9502936 and INT 9802694. 